\title{
Méthodologie d'aide à l'évaluation des stratégies de réhabilitation après un accident : mise en œuvre et résultats
}

\author{
O.M. BADIE*, Ch. BRUN-YABA*, B. CESSAC*, J.M. PERES*
}

(Manuscrit reçu le 4 janvier 2000, révisé le 29 mars 2000, accepté le 27 juillet 2000)

RÉSUMÉ La gestion post-accidentelle relève d'une démarche d'optimisation, dans un contexte multicritère, s'appuyant aussi bien sur des paramètres quantitatifs que qualitatifs. Afin de répondre à cette problématique, un travail méthodologique a été réalisé et mis en reuvre dans le cadre de l'exercice national de crise Becquerel, qui a simulé un accident de type Borax sur un réacteur expérimental. La méthodologie développée consiste, à partir de l'évaluation systématique d'indicateurs quantifiables, à proposer des éléments d'intercomparaison des diverses stratégies de réhabilitation. Ainsi chaque stratégie est appréciée en termes de gain radiologique pour la population, et d'incidences induites telles que le temps de réalisation, les moyens matériels et humains nécessaires, les doses aux intervenants, ainsi que les volumes de déchets générés. Dans ce contexte, les évaluations ont notamment mis en évidence l'importance des contre-mesures destinées à réduire l'exposition externe qui est la voie d'exposition prépondérante. En réduisant l'activité massique dans les aliments, les contre-mesures relatives aux compartiments menant à une dose par ingestion, présentent un intérêt essentiellement au regard des limites de commercialisation. Ce travail constitue une première contribution utile à la clarification des enjeux liés à la réhabilitation post-accidentelle. Il a également fait apparaître des besoins dans plusieurs domaines dont : la formalisation des contre-mesures afin de les regrouper en une base de données, l'adaptation des outils de calcul en vue d'en accroître la souplesse d'emploi, l'amélioration de la connaissance des aspects opérationnels des interventions.

ABSTRACT Methodology for rehabilitation strategies evaluation aid after an accident: application and results.

Post-accidental management is a matter for an optimisation process in a multicriteria context, which has to combine quantitative parameters as well as qualitative parameters. In order to fit with this problematic, a methodological work has been realised at IPSN, in the context of the Becquerel national exercise, which has simulated a Borax type accident on an experimental reactor. The methodology consists in making a systematic evaluation of indicators in order to provide intercomparating data for many rehabilitation strategies. Thus, each strategy

* Institut de protection et de sûreté nucléaire, DPRE, SERGD/LESTS, BP 6, 92265 Fontenay-aux-Roses Cedex, France. 
is evaluated on the one hand in terms of radiological benefit and on the other hand, in terms of incidences such as duration of realisation, materials and human means, workers doses, amount of generated wastes. In this context, calculations highlighted the importance of counter-measures aiming at reducing the external exposure, which is the dominating exposure pathway. The counter-measures aimed at reducing the dose by ingestion lead to a reduction of specific activity are then useful mainly in regard to the limits of commercialisation. This work is a first and useful contribution to the clarification of the post-accidental rehabilitation problem. It has also put the light on three important needs: formalisation of counter-measures and gathering into a data base, adaptation of calculation tools to make their use easier, and improvement of the knowledge of the operational considerations for the interventions.

\section{Introduction}

La gestion d'un accident nucléaire peut se décliner en deux phases: une phase de crise, où des décisions urgentes visant à protéger les populations doivent être prises, et une phase post-crise dite « post-accidentelle » qui vise à rétablir des conditions de vie acceptables pour les populations touchées. La première phase commence dès le début de l'accident. Elle peut se poursuivre pendant plusieurs jours voire plusieurs semaines. À ce stade, on s'appuie sur des critères d'intervention radiologiques pour décider de l'application de contre-mesures. La seconde phase peut commencer dès les premiers jours après l'accident et se poursuivre pendant quelques mois, voire plusieurs années. Les actions de réhabilitation mises en cuvre au cours de cette phase ont pour objectif d'obtenir une situation nouvelle, suffisamment stable, et jugée acceptable sur les plans sanitaire, économique et psychosociologique par les diverses parties prenantes. L'obtention de cette situation est donc sous-tendue par des choix complexes qui s'appuient sur des informations de nature très différente, mais qui sont toutes significatives dans la prise de décision. La gestion post-accidentelle ne relève donc plus d'une logique d'intervention, mais d'une démarche d'optimisation dans un contexte multicritère, et qui nécessite la manipulation d'une grande quantité de données relatives à l'accident, à l'environnement et aux contre-mesures.

Afin de répondre à cette problématique, une méthodologie a été développée et mise en œuvre dans le cadre de l'exercice interministériel de sécurité nucléaire Becquerel joué en octobre 1996 (Crabol et al., 1998), dont les objectifs étaient d'une part d'évaluer la capacité de gestion des structures opérationnelles dans leur rôle (prise de décision, intervention, expertise, communication), et d'autre part, d'étudier les conditions de circulation de l'information relative à l'accident. Cet exercice a comporté deux phases, la première dite du «plan particulier d'intervention» (PPI), la seconde dite du «plan d'actions post-accidentelles» (PPA). La méthodologie consiste, à partir de l'évaluation systématique d'indicateurs quantifiables, à proposer des éléments d'intercomparaison de diverses stratégies de réhabilitation. Elle s'appuie sur quatre étapes, qui sont décrites et illustrées plus précisément dans les paragraphes suivants : 
- la première étape correspond à un état des lieux, c'est-à-dire à une description de l'environnement contaminé ;

- la seconde consiste à calculer l'évolution temporelle de la dose aux populations sans contre-mesure, ainsi que la contribution de chacun des compartiments à cette dose. On évalue également les niveaux de contamination dans les sols et dans les denrées alimentaires ;

- la troisième, correspond au choix d'une contre-mesure. Elle suppose donc un prérequis qui est une base de données détaillée des différentes contre-mesures disponibles;

- la dernière étape, itérative avec la précédente, consiste à apprécier le gain radiologique et les incidences induites pour les contre-mesures puis pour les stratégies sélectionnées.

\section{Caractérisation de l'environnement}

\subsection{Caractéristiques du dépôt}

L'exercice Becquerel (Crabol et al., 1998) simule un accident de type BORAX (fusion totale et immédiate du cœur sous eau) sur un réacteur expérimental du centre d'études de Saclay. Dans le cadre de cette étude une surface contaminée d'environ $16 \mathrm{~km}^{2}$ est étudiée, dans laquelle sont considérés les trois radionucléides $\left({ }^{131} \mathrm{I},{ }^{134} \mathrm{Cs}\right.$ et ${ }^{137} \mathrm{Cs}$ ) qui constituent l'essentiel des dépôts. Étant données l'imprécision des mesures de dépôts, l'hétérogénéité des retombées, et l'incertitude liée aux calculs, il est préférable d'établir des zones d'isoconcentration (ou isodépôt) différant d'environ un facteur 10. Ainsi, on distingue trois zones de contamination différentes (Tab. I).

\section{TABLEAU I}

Caractéristiques des zones contaminées.

Caracteristics of contaminated zones.

\begin{tabular}{|c|c|c|c|c|c|c|c|}
\hline zones & $\begin{array}{c}{ }^{134} \mathrm{Cs} \\
\left(\mathrm{Bq} \mathrm{m}^{-2}\right)\end{array}$ & $\begin{array}{c}{ }^{137} \mathrm{Cs} \\
\left(\mathrm{Bq} \mathrm{m}^{-2}\right)\end{array}$ & $\begin{array}{c}{ }^{131} \mathbf{I} \\
\left(B q \mathbf{m}^{-2}\right)\end{array}$ & $\begin{array}{c}\text { Superficie } \\
\text { totale } \\
\text { (ha) }\end{array}$ & $\begin{array}{l}\text { Superficie } \\
\text { espace } \\
\text { (ha) }\end{array}$ & $\begin{array}{c}\text { Superficie } \\
\text { zone agricole } \\
\text { (ha) }\end{array}$ & $\begin{array}{c}\text { Population } \\
\text { (nombre } \\
\text { d'habitants) }\end{array}$ \\
\hline zone I & 10000 & 24000 & 1512000 & 98 & 31 & 52 & $\begin{array}{l}\quad 400 \\
\text { (les travailleurs du } \\
\text { site de Saclay ne } \\
\text { sont pas comptés) }\end{array}$ \\
\hline zone II & 1250 & 3000 & 189000 & 740 & 450 & 200 & 18000 \\
\hline zone III & 208 & 500 & 31500 & 750 & 450 & 200 & 25000 \\
\hline
\end{tabular}




\subsection{Caractérisation géographique}

L'environnement au sens géographique, est décomposé en milieux, puis en compartiments. Dans notre étude, les milieux considérés sont le milieu «agricole»et le milieu «espaces bâtis », constitué principalement du milieu urbain. Leur superficie est donnée dans le tableau I. Les milieux forestiers, aquicoles et les grands équipements (tels que les échangeurs autoroutiers) ne sont pas traités ici et feront l'objet d'une étude ultérieure.

Un compartiment est une entité homogène sur le plan de ses caractéristiques (son milieu d'appartenance, sa nature, son usage) et de sa contamination. Il s'agit d'une entité élémentaire, dont on peut évaluer la contribution à l'impact radiologique, aussi bien en termes dosimétriques qu'en termes d'activité. Enfin, un compartiment est également défini comme une cible potentielle élémentaire d'une contremesure, et en ce sens, la définition des compartiments dépend étroitement des contre-mesures disponibles. Dans le cas présent, les milieux et les compartiments sont définis et caractérisés à partir de données de l'Institut géographique national (IGN), d'informations provenant de l'Institut national de la statistique et des études économiques (INSEE), et enfin du PGSE (présentation générale de sûreté de l'établissement) du centre d'études de Saclay (CEA, 1996). Les compartiments étudiés sont les suivants :

- pour le milieu «agricole », il s'agit de la partie aérienne des végétaux facilement récoltables par des moyens mécaniques (végétaux de type céréales et grandes cultures, voire végétaux des zones en friche), des cultures légumières, des sols agricoles, en différenciant les surfaces « sols de champs nus et prairies » des surfaces « sols céréales et grandes cultures », des vignes et vergers, du lait et des produits dérivés, des volailles et œufs, et enfin de la viande,

- pour le milieu « espaces bâtis », ce sont les toits de maisons individuelles, appelés « toitures » dans la suite de l'article, les façades des bâtiments, les rues et trottoirs, les sols des espaces verts, et les arbres et buissons.

\subsection{Caractérisation démographique}

Afin d'évaluer la contribution de chaque compartiment à la dose au public, il est nécessaire de caractériser les interactions du public avec les compartiments. Ces interactions se traduisent, d'une part en temps de présence dans les compartiments ou associations de compartiments, induisant une exposition externe et une exposition interne par inhalation et ingestion de poussières, et d'autre part, en ration alimentaire et degré d'autarcie, pour les compartiments menant à une contamination interne par ingestion (Preziosi et al., 1998).

La taille des populations de chaque zone est donnée dans le tableau I. Leur typologie, retenue pour la définition des groupes de référence pris en compte dans l'étude, a été obtenue en croisant les catégories socioprofessionnelles 
définies dans les statistiques de l'INSEE (Bertrand, 1993; INSEE, 1993) en fonction de la zone d'habitation (milieu rural ou urbain), avec d'une part le type d'habitation (maison individuelle ou logement collectif) et d'autre part un découpage de la population française en classes d'âge (Cessac, 1998). Les données de l'INSEE utilisées ici étant nationales, il est possible d'effectuer cette caractérisation sur l'ensemble du territoire. Cinq groupes ont été retenus comme étant les plus représentatifs des différentes configurations d'exposition. Ces groupes sont les suivants :

- adulte agricole, vivant en zone rurale, dans un habitat individuel ;

- adulte non agricole, vivant en zone urbaine, dans un habitat individuel ;

- adulte non agricole, vivant en zone urbaine, dans un habitat collectif ;

- enfant, vivant en zone urbaine, dans un habitat individuel ;

- nourrisson, supposé avoir un mode de vie unique, quels que soient la zone d'habitation et le mode de vie des parents.
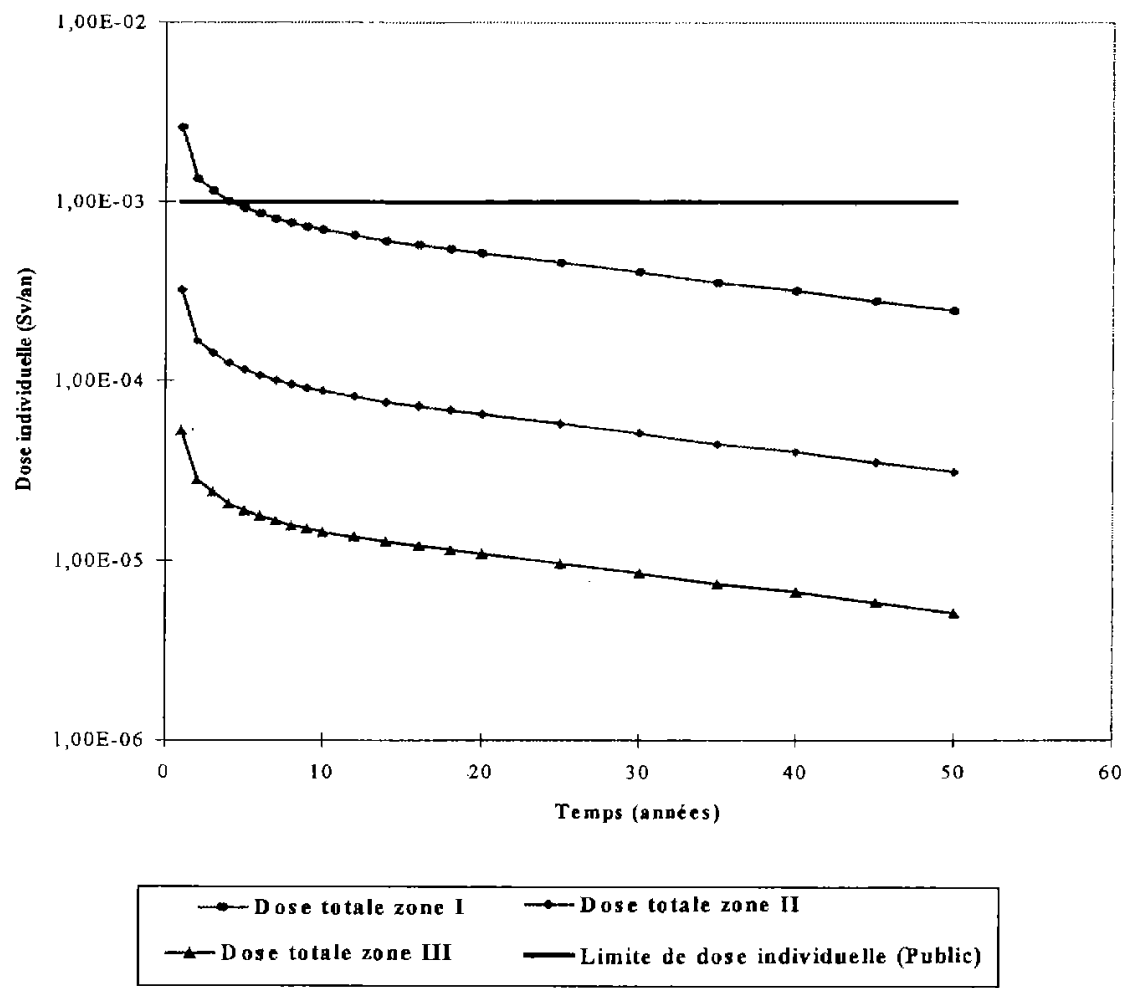

Fig. 1. - Évolution de la dose au cours du temps pour un individu agricole sans contre-mesure.

Change in dose over time for a farm worker with no counter-measure. 


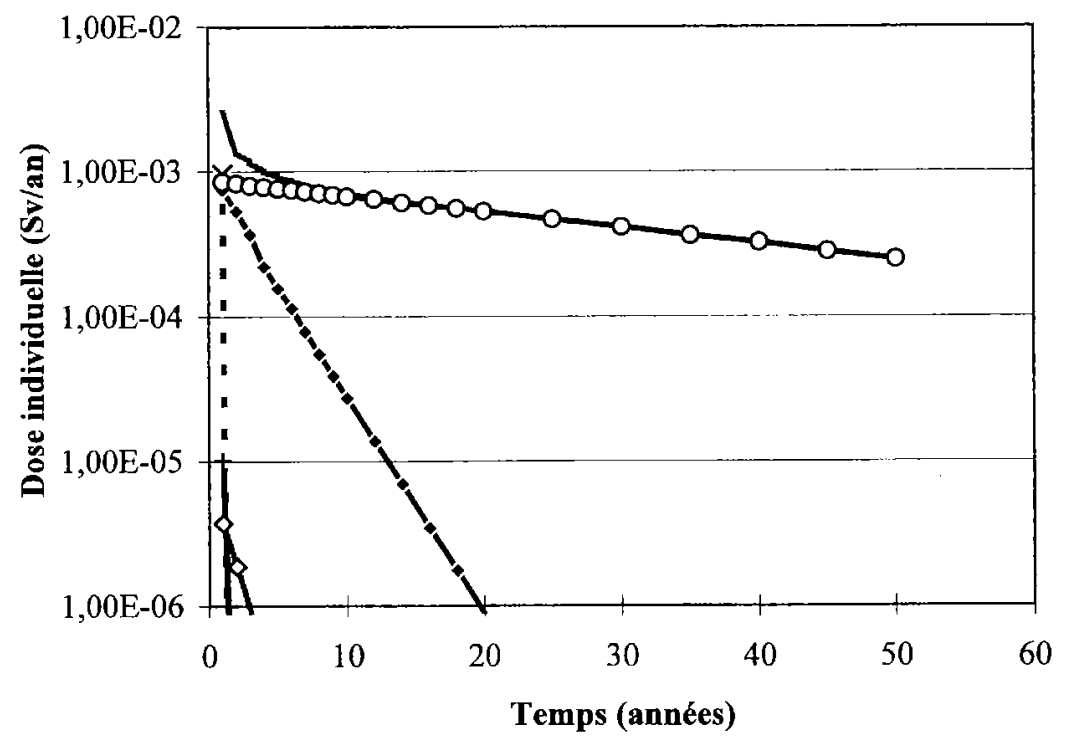

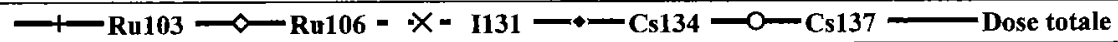

Fig. 2. - Contributions des différents radionucléides à la dose totale un adulte agricole, sans contremesure.

Contributions of the radionuclides to the total dose for a farm worker, with no counter-measure.

\section{Diagnostic de la situation}

\section{1. Évaluation de la dose}

L'impact radiologique post-accidentel est évalué par un calcul de dose efficace annuelle. Par convention, le terme « dose » sera employé dans la suite de l'article pour désigner cette dose efficace annuelle. Les calculs sont réalisés par les codes ASTRAL (Maubert et al., 1997) et ABRICOT (Santucci, 1995 ; Klos et al., 1999) qui modélisent le transfert des radioéléments du terme source à l'homme à travers la biosphère décrite par un ensemble de compartiments. Les doses sont calculées pour chaque groupe de population, sur chacune des trois zones de contamination considérées, sur une période de 50 années suivant l'accident, et pour les trois 
radionucléides rejetés. Les résultats exposés ne considèrent pas les doses dues au passage du panache, qui sont supérieures d'un facteur 3 à 4 à celles induites par le dépôt lors de la première année. La figure 1 présente l'évolution de la dose que pourrait recevoir un adulte agricole. Il s'agit du groupe dont le mode de vie est le plus pénalisant, en raison de son degré d'autarcie et du temps qu'il passe à l'extérieur des bâtiments. La figure 2 présente les contributions des différents radionucléides à la dose d'un adulte agricole, et leurs évolutions dues à la décroissance radioactive.

Les résultats des tableaux II et III montrent que l'exposition externe, dans le cadre de cette étude, représente la source d'exposition majeure avec, la première année, une contribution à la dose totale de 88 à $100 \%$, sauf dans le cas des adultes agricoles pour qui elle ne représente que $31 \%$ de la dose totale, contre $69 \%$ pour l'ingestion. Au cours du temps, cette prédominance s'accentue pour atteindre, dès la deuxième année, une contribution supérieure à $82 \%$, quel que soit le groupe de référence considéré. Dans tous les cas, l'inhalation est une voie d'exposition négligeable.

\section{TABLEAU II}

Contributions relatives des différentes voies d'exposition à la dose totale à $\mathbf{1}$ an après l'accident. Relative contributions of different exposure pathways to total dose one year after the accident.

\begin{tabular}{|l|c|c|c|c|c|c|}
\hline & & $\begin{array}{r}\text { Adulte } \\
\text { agricole }\end{array}$ & $\begin{array}{c}\text { Adulte urbain } \\
\text { Habitat } \\
\text { individuel }\end{array}$ & $\begin{array}{c}\text { Enfant urbain } \\
\text { Habitat } \\
\text { individuel }\end{array}$ & $\begin{array}{c}\text { Adulte urbain } \\
\text { Habitat } \\
\text { collectif }\end{array}$ & Nourrisson \\
\hline $\begin{array}{l}\text { Dose annuelle } \\
\text { (mSv) }\end{array}$ & 3.3 & 1.1 & 1.2 & 0.7 & 0.4 \\
\hline $\begin{array}{l}\text { Contribution des } \\
\text { voies d'exposition } \\
\text { à la dose totale }\end{array}$ & Exp. Ext. & $31 \%$ & $97 \%$ & $88 \%$ & $100 \%$ & $100 \%$ \\
\hline
\end{tabular}

\section{TABLEAU III}

Contributions relatives des différentes voies d'exposition à la dose totale à $\mathbf{2}$ ans après l'accident. Relative contributions of different exposure pathways to total dose two years after the accident.

\begin{tabular}{|l|c|c|c|c|c|c|}
\hline & & $\begin{array}{c}\text { Adulte } \\
\text { agricole }\end{array}$ & $\begin{array}{c}\text { Adulte urbain } \\
\text { Habitat } \\
\text { individuel }\end{array}$ & $\begin{array}{c}\text { Enfant urbain } \\
\text { Habitat } \\
\text { individuel }\end{array}$ & $\begin{array}{c}\text { Adulte urbain } \\
\text { Habitat } \\
\text { collectif }\end{array}$ & Nourrisson \\
\hline $\begin{array}{l}\text { Dose annuelle } \\
\text { (mSv) }\end{array}$ & 0.7 & 0.6 & 0.6 & 0.4 & 0.2 \\
\hline $\begin{array}{l}\text { Contribution des } \\
\text { voies d'exposition }\end{array}$ & $\begin{array}{l}\text { Exp. Ext. : } \\
\text { Ingestion : }\end{array}$ & $82 \%$ & $98 \%$ & $98 \%$ & $100 \%$ & $100 \%$ \\
\hline à la dose totale & Inhalation : & $<1 \%$ & $2 \%$ & $2 \%$ & $0 \%$ & $0 \%$ \\
\hline
\end{tabular}


En ce qui concerne l'exposition externe, les résultats présentés dans le tableau IV montrent que ce sont les compartiments des espaces bâtis, et plus particulièrement les routes et trottoirs, ainsi que les arbres et buissons, qui induisent la majeure partie de la dose, quel que soit le groupe considéré.

\subsection{Activité massique de quelques denrées alimentaires}

Le calcul des activités massiques de chaque radionucléide, dans les compartiments relatifs aux denrées alimentaires complètent les évaluations de dose. Dans le cadre des hypothèses de cette étude, lors de la première année, les limites de concentrations admissibles en ${ }^{131}$ I pour la commercialisation des aliments, sont dépassées pour tous les produits considérés provenant de la zone I, c'est-à-dire jusqu'à deux à trois kilomètres de l'installation (Tab. V). Pour les autres zones étudiées, seul le

\section{TABLEAU IV}

Contribution des compartiments à l'exposition externe, pour un adulte agricole.

Contribution of compartments to external exposure, for an adult farm worker.

\begin{tabular}{|c|c|c|c|c|c|c|c|c|}
\hline \multirow[t]{2}{*}{ an } & & \multirow[t]{2}{*}{$\begin{array}{l}\text { surfaces } \\
\text { agricoles }\end{array}$} & \multicolumn{2}{|c|}{$\begin{array}{l}\text { routes, trottoirs, murs } \\
\text { vitres }\end{array}$} & \multirow[t]{2}{*}{ toits } & \multicolumn{2}{|c|}{$\begin{array}{l}\text { jardins publics, stades, } \\
\text { jardins particuliers }\end{array}$} & \multirow[t]{2}{*}{ tota: } \\
\hline & & & extérieur & intérieur & & sol & arbres, buissons & \\
\hline \multirow[t]{2}{*}{1} & $\mathrm{mSv} / \mathrm{an}$ & 0.2 & \multicolumn{2}{|c|}{0.3} & 0.2 & 0 & 0.3 & 1.0 \\
\hline & & & 0.2 & $7.8 \times 10^{-2}$ & & & & \\
\hline \multirow[t]{2}{*}{2} & $\mathrm{mSv} / \mathrm{an}$ & 0.1 & \multicolumn{2}{|c|}{0.2} & 0.1 & 0 & 0.2 & 0.6 \\
\hline & & & 0.1 & $5.2 \times 10^{-2}$ & & & & \\
\hline \multirow[t]{2}{*}{10} & $\mathrm{mSv} / \mathrm{an}$ & $5.0 \times 10^{-2}$ & \multicolumn{2}{|c|}{$8.2 \times 10^{-2}$} & $6.8 \times 10^{-2}$ & 0 & $8.3 \times 10^{-2}$ & 0.3 \\
\hline & & & $6.1 \times 10^{-2}$ & $2.1 \times 10^{-2}$ & & & & \\
\hline
\end{tabular}

\section{TABLEAU V}

Concentration maximale dans les différents aliments, et dose par ingestion associée pour un adulte agricole - Cas de $\mathbf{l}^{131} I$ en zone $I$.

Maximum concentrations in different foodstuffs and corresponding doses through ingestion for an adult farm worker - Example of ${ }^{131} I$ in zone $I$.

\begin{tabular}{|c|c|c|c|c|c|c|c|}
\hline an & & légumes & céréales & $\begin{array}{c}\text { lait, } \\
\text { prod. déri. }\end{array}$ & $\begin{array}{l}\text { viande de } \\
\text { boucherie }\end{array}$ & $\begin{array}{c}\text { yolaille, } \\
\text { cuffs }\end{array}$ & total \\
\hline 1 & $\mathrm{~Bq} \mathrm{~kg}{ }^{-1}$ & 4600 & $1.2 \times 10^{4}$ & $1.2 \times 10^{5}$ & 2160 & 4500 (ceufs) & \\
\hline (normes de & & $(2000)$ & $(2000)$ & $(500)$ & $(2000)$ & $(2000)$ & \\
\hline commercialisation) & dose $\mathrm{mSv} / \mathrm{an}$ & 0.2 & 0 & 1.9 & $7.3 \times 10^{-3}$ & $1.2 \times 10^{-2}$ & 2.1 \\
\hline \multirow[t]{2}{*}{2} & $\mathrm{~Bq} \mathrm{~kg}^{-1}$ & traces & traces & traces & traces & traces & \\
\hline & dose $\mathrm{mSv} / \mathrm{an}$ & $<10^{-10}$ & 0 & $<10^{-10}$ & $<10^{-10}$ & $<10^{-10}$ & $<10^{-10}$ \\
\hline \multirow[t]{2}{*}{10} & $\mathrm{~Bq} \mathrm{~kg}^{-1}$ & 0 & 0 & 0 & 0 & 0 & \\
\hline & dose $\mathrm{mSv} / \mathrm{an}$ & 0 & 0 & 0 & 0 & 0 & 0 \\
\hline
\end{tabular}


lait dépasse ces limites de commercialisation. Au-delà de la première année, tout en restant inférieur aux limites de commercialisation, le ${ }^{137} \mathrm{Cs}$ peut atteindre, voire dépasser la centaine de $\mathrm{Bq}^{-1}$ dans les viandes.

\section{Sélection d'une contre-mesure}

L'arsenal des contre-mesures disponibles étant vaste, une première sélection a été réalisée à partir essentiellement d'études relatives à l'accident de Tchernobyl (Besnus et al., 1996 ; CCE, 1991, 1996). Les contre-mesures encore au stade de développement ou celles qui semblent inadaptées à une application à grande échelle ont été éliminées. Le tableau VI dresse la liste des types de contre-mesures considérées, associées à leurs compartiments cibles. À chaque type de contre-mesure peut correspondre un ensemble de techniques, réalisées avec des matériels différents. Le décapage des sols/prairies par exemple peut s'effectuer par bulldozer, pelleteuse ou décapeuse.

Une fois qu'une technique a été sélectionnée, ses modalités d'application doivent être précisées : le délai de mise en œuvre de la contre-mesure, sa durée de réalisation, sa répétition sur un même compartiment, sa combinaison avec d'autres contre-mesures et la chronologie de leur mise en œuvre, sont autant d'éléments à considérer. Une stratégie de réhabilitation est donc définie sur la base d'une combinaison de contre-mesures, mais aussi par ces informations de répétitivité, d'ordonnancement et de délai de mise en œuvre. Ainsi, par exemple, dans le milieu urbain, le compartiment « rues et trottoirs » peut être balayé, lavé ou décapé. Le lavage et le balayage peuvent être répétés, et les techniques peuvent être combinées (Fig. 3).

TABLEAU VI

Liste des contre-mesures étudiées.

List of counter-measures studied.

\begin{tabular}{|l|l|l|}
\hline \multicolumn{1}{|c|}{ milieu } & \multicolumn{1}{|c|}{ compartiment } & \multicolumn{1}{c|}{ types de contre-mesures } \\
agricole & champs/prairies & labour, décapage \\
& grandes cultures et céréales (sols) & labour, décapage \\
& grandes cultures et céréales (végétation) & moisson, arrachage (bulldozer) \\
urbain & rues et trottoirs & lavage \\
& façades (murs et vitres) & lavage \\
& toits maisons (pentus) & lavage, échange toiture \\
& sols des espaces verts & décapage \\
& arbres et buissons & élagage \\
\hline
\end{tabular}




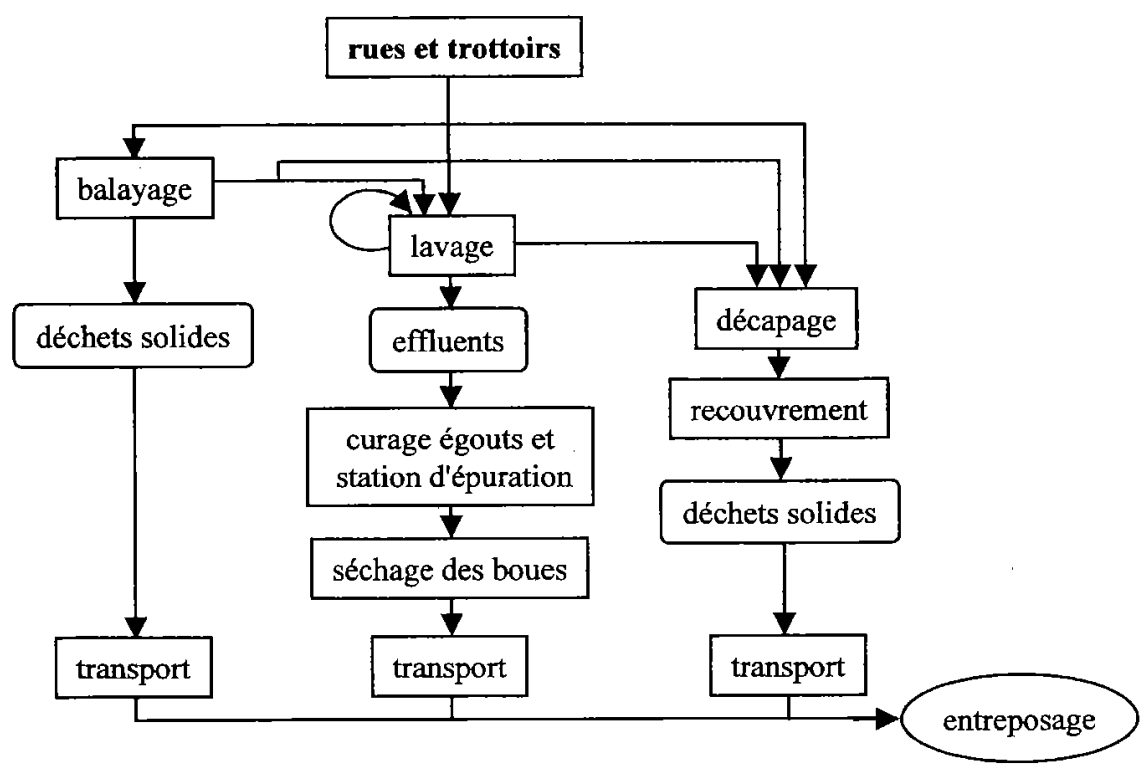

Fig. 3. - Techniques potentielles pour le compartiment « routes et trottoirs ».

Potential techniques for * roads and pavement * compartment.

\section{5. Évaluation des stratégies}

L'évaluation de la performance d'une stratégie consiste à calculer les indicateurs de gains et d'incidences liés à l'application d'une contre-mesure, puis à intégrer les résultats propres à la contre-mesure à ceux de la stratégie en cours d'élaboration. Les indicateurs retenus doivent être accessibles et représentatifs des stratégies. Dans cette application, les gains des stratégies sont les doses aux groupes de population retenus, et les incidences sont les doses aux intervenants, le temps de réalisation des stratégies, ainsi que les moyens humains et matériels à mettre en ouvre.

\subsection{Les gains radiologiques}

\subsubsection{Milieu agricole}

Le gain radiologique des contre-mesures sélectionnées est donné dans le tableau VII, pour ce qui concerne le ${ }^{137} \mathrm{Cs}$, et la zone I. Le labour des sols nus ou dont la 
TABLEAU VII

Effet de différentes contre-mesures sur des compartiments agricoles la première année Cas du ${ }^{137} \mathrm{Cs}$, zone $\mathrm{I}$.

Effect of the various counter-measures on rural compartments during first year Example of ${ }^{137} \mathrm{Cs}$ in zone $\mathrm{I}$.

\begin{tabular}{|c|c|c|c|c|c|c|c|c|}
\hline \multirow{2}{*}{$\begin{array}{c}\text { Nature de la } \\
\text { contre-mesure } \\
\begin{array}{c}\text { Compartiment } \\
\text { intéressé }\end{array}\end{array}$} & contr & ans & \multicolumn{2}{|c|}{ Sols agricoles nus on de prairie } & & bour & \multicolumn{2}{|c|}{$\begin{array}{l}\text { Cultures céréalières } \\
\text { à maturité } \\
\text { Moisson ou ensilage } \\
\text { + Labour }\end{array}$} \\
\hline & Sol & végétaux & Sol & végétaux & Sol & végétaux & Sol & végétaux \\
\hline $\mathrm{Bq} \mathrm{kg}^{-1}$ & 1700 & 120 & 57 & 4,2 & 5,7 & 0,4 & 11 & 0.8 \\
\hline
\end{tabular}

végétation est peu développée, conduit à la réduction jusqu'à un facteur 30 des concentrations d'activité dans l'herbe, et par voie de conséquence dans le lait et dans la viande. Le décapage des sols permet une réduction des concentrations d'activité dans les aliments supérieure d'environ un facteur 10 à celle due à un labour. Enfin la récolte de végétaux en préalable à toute contre-mesure représente une réduction de la contamination ultérieure des sols qui peut aller jusqu'à un facteur 5 . Ce genre de contre-mesure semble parfaitement adapté aux grandes cultures, de type céréalière, lorsque leur degré de maturation est avancé.

L'influence sur la dose des stratégies étudiées en milieu agricole reste très limitée dans la mesure où l'exposition externe par les sols de culture et l'exposition interne due à l'ingestion d'aliments provenant de ces sols, sont des voies secondaires dans la dose totale (Tabs. IV et V). Par contre, ces stratégies conservent toute leur importance en terme de réduction des activités massiques au regard des limites de commercialisation des aliments.

\subsubsection{Milieu urbain}

Les contre-mesures sélectionnées pour le milieu urbain permettent de réduire considérablement l'exposition externe du public. Une stratégie combinant les contremesures les plus efficaces (lavages successifs et élagage) peut conduire à une réduction de la dose d'un facteur 3 (Fig. 4).

L'élagage des arbres et des buissons entraine une réduction de la dose totale d'environ un facteur 1,5. L'intérêt de cette contre-mesure devra être précisé ultérieurement en fonction des saisons, donc de l'état de la végétation, en distinguant les arbres des buissons. 
Dose annuelle (mSv/an)

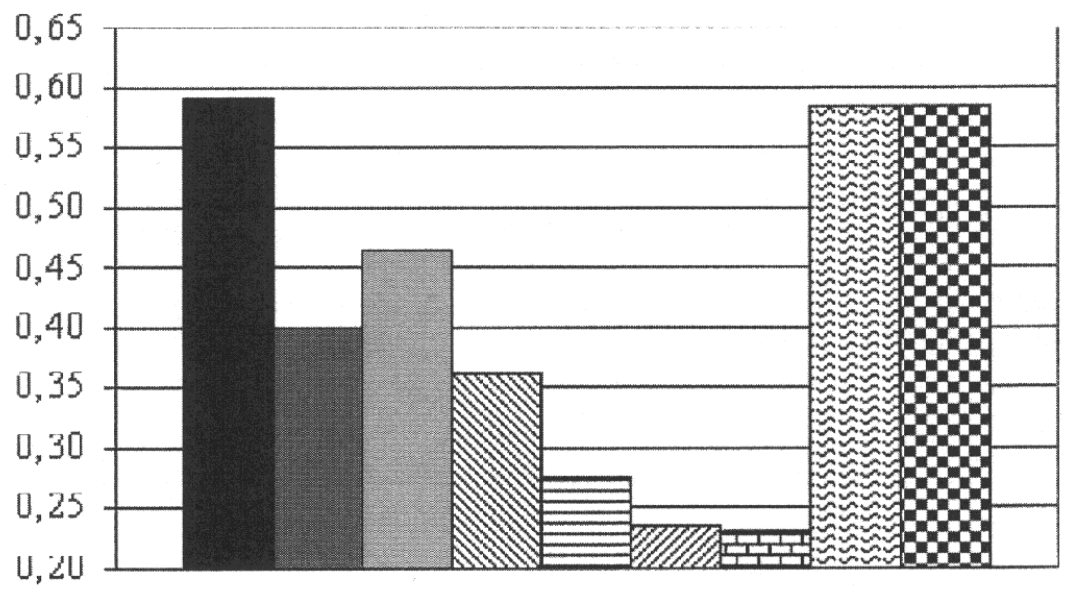

a Hors CM

- Lavage à $t=1$ semaine

$\square$ Lavage à $\mathrm{t}=1$ mois

Lavages successifs au cours du $1^{\mathrm{er}}$ mois

日 Lavages successifs + changements des toits

Z Lavages successifs + élagage des arbres et buissons

$\square$ Lavages successifs + élagage des arbres et buissons + décapage des jardins potagers

v Labour

Décapage des surfaces agricoles + labour

Fig. 4. - Comparaison de l'incidence de différentes stratégies sur l'exposotion d'un enfant urbain (habitat individuel), lors de la première année après l'accident, zone 1.

Comparison of effects of the various strategies on exposure of an urban child (private house) in the first year after the accident, zone 1 .

En revanche, le changement des toitures de l'habitat individuel n'induit pas de gain radiologique significatif par rapport à des lavages successifs. De plus, les contraintes logistiques associées à ce type de contre-mesures paraissent relativement lourdes.

La figure 4 illustre également la complexité du système: à partir d'une seule contre-mesure, comme le lavage, il est possible d'obtenir des stratégies aux résultats très différents selon le délai de mise en œuvre de la contre-mesure, et sa répétition. Ainsi le lavage des trottoirs, des routes et des toitures induit une réduction de la dose d'un facteur 1 à 2 en fonction du délai d'intervention. 


\subsection{Les incidences des interventions}

Les incidences évaluées sont exprimées en dose individuelle aux intervenants, en temps de réalisation, et en moyens humains et matériels nécessaires. Ces paramètres sont très liés les uns aux autres : la dose aux intervenants est calculée à partir d'une dose horaire, et du temps de réalisation de la contre-mesure. La dose horaire est obtenue par la définition du poste de travail qui est lui-même fonction de la technique. Le temps de réalisation quant à lui, dépend de la vitesse d'exécution de la technique, de la taille du compartiment à traiter, mais également de la quantité des moyens engagés. D'autres paramètres, reflétant les aspects socio-économiques, psychosociologiques, ou tels que les effets secondaires des contre-mesures, ne sont pas étudiés dans cette application.

Par hypothèse dans cette étude, la durée des stratégies ne doit pas excéder une année, soit $2000 \mathrm{~h}$ de travail. Au-delà, des équipes supplémentaires sont mises à contribution. Les résultats obtenus correspondent donc à la mise en œuvre de moyens minimaux pour réaliser les contre-mesures en moins d'une année, et donc à une dose maximale pour les intervenants.

Les gains et les incidences de chaque stratégie sont fournis dans des tableaux récapitulatifs tels que les tableaux VIII et IX. Ces tableaux pourraient être considérés comme la trame de fiches d'aide à la décision qui seraient fournies aux décideurs.

Les résultats montrent que la dose aux intervenants est faible quelle que soit la zone considérée, et n'apparaît pas comme un paramètre dimensionnant dans le cas spécifique de cette étude. Ainsi, que ce soit en milieu agricole ou en milieu urbain, la dose maximale reçue par les individus en charge de la réalisation des contremesures est toujours inférieure à $0,1 \mathrm{mSv}$, bien que seuls des moyens sans protection particulière aient été considérés. II apparaît également que les doses aux intervenants dans les zones II et III sont le plus souvent inférieures à celles des intervenants de la zone I, du fait de la différence des niveaux de contamination entre les zones, et de la taille des compartiments à traiter qui impose une multiplication des moyens pour rester en dessous d'une année de travail. Il existe cependant, des contre-mesures telles que le labour ou le lavage, qui se réalisent en un temps suffisamment court pour ne pas nécessiter de moyens différents quelle que soit la zone traitée. Dans ce cas, les doses induites aux intervenants peuvent être supérieures en zone II et III de par l'étendue des surfaces.

La durée de réalisation des stratégies qui comprennent un décapage ou un changement des toits en revanche, est considérablement plus importante, et pour respecter les hypothèses de l'étude, elles requièrent plusieurs centaines de personnes en zones II et III. Enfin, les stratégies qui mettent en cuvre un retrait du support contaminé (décapage de sols, changement de toitures...) produisent des déchets dont la gestion impose de fortes contraintes liées aux opérations de transport, de traitement et de 


\section{TABLEAU VIII}

Intercomparaison des stratégies du milieu urbain dans la zone I, première année. Comparison of strategies in an urban milieu in zone I, first year.

\begin{tabular}{|c|c|c|c|c|c|c|}
\hline $\begin{array}{l}\text { ZONE I } \\
\text { popul. } 400 \text { pers. }\end{array}$ & $\begin{array}{l}\text { stratégie } \\
1 . a\end{array}$ & $\begin{array}{c}\text { stratégie } \\
1 . \mathrm{b}\end{array}$ & $\begin{array}{c}\text { stratégie } \\
\text { 1.c }\end{array}$ & $\begin{array}{c}\text { stratégie } \\
2\end{array}$ & $\begin{array}{c}\text { stratégie } \\
3\end{array}$ & $\begin{array}{l}\text { stratégie } \\
4\end{array}$ \\
\hline $\begin{array}{l}\text { rues/trottoirs/ } \\
\text { façades/toits }\end{array}$ & $\begin{array}{l}1 \text { lavage } \\
\text { immédiat }\end{array}$ & $\begin{array}{l}\text { lavages } \\
\text { successifs } \\
\text { immédiats }\end{array}$ & $\begin{array}{l}\text { lavage } \\
\text { à } 1 \text { mois }\end{array}$ & $\begin{array}{l}\text { lavages } \\
\text { successifs } \\
\text { immédiats }\end{array}$ & $\begin{array}{c}\text { lavages } \\
\text { successifs } \\
\text { immédiats }\end{array}$ & $\begin{array}{c}\text { lavages } \\
\text { successifs } \\
\text { immédiats }\end{array}$ \\
\hline jard. publics/stades & & & & & décapage & \\
\hline arbres & & & & élagage & élagage & \\
\hline toits & & - & & & & changement \\
\hline moyens humains & 4 & 4 & 4 & 10 & 12 & 10 \\
\hline engin de lavage & 2 & 2 & 2 & 2 & 2 & 2 \\
\hline camion grumier & & & & 1 & 1 & \\
\hline camion transport & & & & 2 & 4 & 4 \\
\hline bulldozer & & & & & 1 & \\
\hline dose intervenants (mSv) & $10^{-4}$ & $10^{-3}$ & $10^{-5}$ & $10^{-5}$ à $10^{-2}$ & $10^{-5}$ à $10^{-2}$ & $10^{-5}$ à $10^{-2}$ \\
\hline temps de réalisation (h) & 3. & 10 & 3 & 1250 & 1250 & 2000 \\
\hline & \multicolumn{6}{|c|}{ Déchets (en tonnes) } \\
\hline végétal & & & & 650 & 650 & 600 \\
\hline minéral & & & & & 62000 & \\
\hline & \multicolumn{6}{|c|}{ Dose population (mSv) } \\
\hline $\begin{array}{c}\text { Hab. collect. }(130 \text { pers }) \\
\text { sans C.M : } 0,7\end{array}$ & 0,55 & 0,5 & 0,6 & 0,3 & 0,2 & 0,5 \\
\hline $\begin{array}{c}\text { Hab. indivi. (160 pers) } \\
\text { sans C.M : } 1,2\end{array}$ & 0,8 & 0,7 & 1 & 0,45 & 0,4 & 0,6 \\
\hline
\end{tabular}

stockage. Selon la nature et la quantité des déchets, ceux-ci pourront subir une réduction de volume, être stabilisés ou stockés directement. Plusieurs filières sont envisageables, et seront étudiées ultérieurement. Dans cette étude, au vu des activités des déchets potentiellement générés, un stockage direct en décharge de type TFA (décharge recevant des déchets très faiblement radioactifs) semble une solution appropriée sur le plan radiologique au moins pour les déchets non organiques. Il sera naturellement nécessaire de considérer la faisabilité industrielle, les effets secondaires ainsi que les incidences économiques d'une mise en décharge directe avant d'adopter définitivement cette solution. 


\section{TABLEAU IX}

Intercomparaison des stratégies du milieu urbain dans la zone III, première année.

Comparison of strategies in an urban milieu in zone III, first year.

\begin{tabular}{|c|c|c|c|c|c|c|}
\hline $\begin{array}{l}\text { ZONE I } \\
\text { popul. } 25000 \text { pers. }\end{array}$ & $\begin{array}{l}\text { stratégie } \\
\text { 1.a }\end{array}$ & $\begin{array}{c}\text { stratêgie } \\
1.6\end{array}$ & $\begin{array}{c}\text { stratégie } \\
1.0\end{array}$ & $\begin{array}{c}\text { stratégie } \\
2 \\
\end{array}$ & $\begin{array}{l}\text { strategie } \\
3\end{array}$ & $\begin{array}{l}\text { stratégie } \\
4\end{array}$ \\
\hline $\begin{array}{l}\text { rues/trottoirs/ } \\
\text { façades/toits }\end{array}$ & $\begin{array}{l}1 \text { lavage } \\
\text { immédiat }\end{array}$ & $\begin{array}{l}\text { lavages } \\
\text { successifs } \\
\text { immédiats }\end{array}$ & $\begin{array}{l}\text { lavage } \\
\text { à } 1 \text { mois }\end{array}$ & $\begin{array}{c}\text { lavages } \\
\text { successifs } \\
\text { immédiats }\end{array}$ & $\begin{array}{l}\text { lavages } \\
\text { successifs } \\
\text { immédiats }\end{array}$ & $\begin{array}{l}\text { lavages } \\
\text { successifs } \\
\text { immédiats }\end{array}$ \\
\hline jard. publics/stades & & & & & décapage & \\
\hline arbres & & & & élagage & élagage & \\
\hline toits & & & & & & changement \\
\hline moyens humains & 4 & 4 & 4 & 50 & 60 & 350 \\
\hline engin de lavage & 2 & 2 & 2 & 2 & 2 & 2 \\
\hline camion grumier & & & & 10 & 10 & \\
\hline camion transport & & & & 18 & 22 & 170 \\
\hline bulldozer & & & & & 2. & \\
\hline dose intervenants (mSv) & $10^{-3}$ & $10^{-3}$ & $10^{-3}$ & $10^{-6}$ a $10^{-3}$ & $10^{-6}$ à $10^{-3}$ & $10^{-6}$ à $10^{-3}$ \\
\hline temps de réalisation (b) & 150 & 450 & 150 & 2000 & 2000 & 2000 \\
\hline & \multicolumn{6}{|c|}{ Déchets (en tonnes) } \\
\hline végétal & & & & 20000 & 20000 & \\
\hline minéral & & & & & 130000 & $4 \times 10^{-4}$ \\
\hline & \multicolumn{6}{|c|}{ Dose population (mSv) } \\
\hline $\begin{array}{l}\text { Hab. collect. (8 } 000 \text { pers) } \\
\text { sans C.M : } 1,4 \times 10^{-2}\end{array}$ & $1,1 \times 10^{-2}$ & $1,0 \times 10^{-2}$ & $1,2 \times 10^{-2}$ & $5,4 \times 10^{-3}$ & $4,6 \times 10^{-3}$ & $1,0 \times 10^{-3}$ \\
\hline $\begin{array}{l}\text { Hab. indivi. (10000 pers) } \\
\text { sans C.M : } 2,2 \times 10^{-2}\end{array}$ & $1,5 \times 10^{-2}$ & $1,9 \times 10^{-2}$ & $1,2 \times 10^{-2}$ & $9,2 \times 10^{-3}$ & $8,4 \times 10^{-3}$ & $1,2 \times 10^{-2}$ \\
\hline
\end{tabular}

Ces premiers résultats confirment que les moyens à mettre en œuvre, la durée de réalisation et la gestion des déchets, peuvent largement pondérer l'intérêt en terme de gains radiologiques de certaines contre-mesures, contre-mesures dont les effets secondaires devront également être considérés. Par exemple, avant de choisir un décapage, il sera indispensable de vérifier l'incidence agronomique de la suppression d'une couche fertile d'une dizaine de centimètres sur des sols agricoles. Ainsi, au changement des toits et au décapage, il peut être préférable de choisir respectivement des lavages successifs et un labour, qui apparaissent comme des opérations efficaces, simples et rapides. 


\section{Conclusion}

Ce travail constitue une première contribution à la clarification des enjeux de la réhabilitation post-accidentelle en proposant une évaluation de stratégies à partir d'indicateurs quantifiables. Cette évaluation doit naturellement être complétée par d'autres indicateurs (psychosociologiques, socio-économiques) nécéssitant l'implication des différents acteurs locaux et nationaux.

Dans le cadre des hypothèses et de la situation retenue liée à l'exercice Becquerel, les évaluations effectuées montrent que l'irradiation externe est la voie d'exposition prépondérante pour la majeure partie de la population la première année, et pour la totalité de la population dès la seconde année, l'ingestion n'étant une voie prépondérante que la première année pour des groupes présentant un fort degré d'autarcie. D'autre part, les compartiments du milieu bâti étant responsables de l'essentiel de l'irradiation externe, les contre-mesures qui lui sont relatives induisent les gains radiologiques les plus significatifs. Par conséquent la prédominance de l'exposition externe dans le milieu bâti conduit à devoir améliorer les outils de calculs dans ce domaine. Enfin, la gestion des déchets, même si elle n'a été que peu abordée dans l'étude, est une opération très pénalisante pour certaines stratégies du fait de la quantité de déchets potentiellement générés dans une zone de quelques kilomètres-carrés.

La méthodologie développée au cours de cette étude offre un cadre organisationnel basé sur la comparaison d'indicateurs, utile pour gérer la complexité de la réhabilitation. Ses principaux objectifs sont d'une part de faciliter les calculs des performances des différentes stratégies au fur et à mesure de leur construction, et d'autre part, d'établir des fiches synthétiques qui traduisent les caractéristiques des stratégies, dans une optique d'aide à la décision. Son application a mis en évidence plusieurs besoins :

- Le premier est de poursuivre la quantification des indicateurs proposés dans le cadre de nouvelles contre-mesures, et de milieux plus ruraux.

- Il serait également très utile de développer une base de données des contre-mesures renseignée de toutes les caractéristiques permettant de calculer les indicateurs. En effet, un nombre important de données sont actuellement disponibles, mais dispersées et non normalisées.

- Cette base de données doit ensuite être associée à un système d'exploitation qui permettrait d'analyser rapidement plusieurs situations d'environnement contaminés en comparant de nombreuses stratégies, de manière systématique.

- Il serait aussi très profitable de disposer d'une base de données descriptives de l'environnement des installation nucléaires, afin de pouvoir caractériser rapidement les groupes de population à considérer, et surtout les milieux et compartiments à traiter.

- Enfin, il sera nécessaire d'affiner la méthodologie, en intégrant les phénomènes de contamination secondaires qui résultent des flux d'activité entre les compartiments. 


\section{ÉVALUATION DES STRATÉGIES DE RÉHABILITATION}

\section{RÉFÉRENCES}

Bertrand M. (1993) Consommation et lieux d'achats des produits alimentaires en 1991. INSEE Résultats, Consommation-Modes de vie $n^{\circ}$ 54-55.

Besnus F., Jouve A., Pérès J.M., Santucci P. (1996) Réhabilitation de surfaces contaminées à la suite d'un accident nucléaire et gestion des déchets qui en sont issus. Rapport IPSN/DPRE $\mathbf{n}^{\circ}$ 96/01.

CCE (1991) International scientific collaboration on the consequences of the Chernobyl accident (199195), Experimental Collaboration Project $n^{\circ} 4$, Strategies of decontamination. Final report EUR 16530.

CCE (1996) Improvement of pratical countermeasures: the urban environment, Post-Chernobyl action. Report EUR 12555

CEA (1996) Présentation générale de sûreté de l'établissement de Saclay. Rapport DCEAS/DIR/96/PGSE/002 Indice A.

Cessac B. (1998) Habitudes alimentaires retenues pour les évaluations post-accidentelles. Note technique DPRE/SERGD/LESTS 98/14. Communication personnelle.

Crabol B., Manesse D., Fache Ph., Linge I., Pavlovski O., Rousseau D. (1998) Dossier de référence de l'exercice Becquerel d'octobre 1996. Communication personnelle.

INSEE (1993) Annuaire statistique de la France - Résultats de 1992. Document INSEE, Vol. 97, nouvelle série $\mathrm{n}^{\circ} 39$.

Klos R.A., Simon I., Bergstrom U., Uijt De Haag A.M., Valentin-Ranc C., Zeevaert T., Reid J.A.K., Santucci P., Titley J., Standby J. (1999). Complementary Studies: Biosphere modelling for dose assessments of radioactive waste repositories, J. Environ. Rad. 42, 237-254.

Maubert H., Renaud P., Bernié J.C., De Drouas G., Fache P., Métivier J.-M. (1997) ASTRAL : un logiciel pour l'évaluation des conséquences d'un rejet accidentel de radionucléides dans l'environnement, Radioprotection 32, 357-368.

Preziosi P., Galan P., Granveau C., Deheeger M., Papoz L., Hercberg S. (1988) Consommation alimentaire d'un échantillon représentatif de la population du Val de Marne : I. Contribution des aliments à l'apport énergétique, Rev. Épidémiol. Santé Pub. 39, 221 - 31.

Santucci P. (1995) Conceptual and mathematical modelling of the biosphere: ABRICOT - version 2.0. Note technique IPSN/DPRE/SERGD 95/04. Communication personnelle. 